PREPARED FOR THE U.S. DEPARTMENT OF ENERGY, UNDER CONTRACT DE-AC02-76CH03073

PPPL-3536

PPPL-3536

UC-70

A Fluid-kinetic Hybrid Electron Model for Electromagnetic Simulations

by

Zhihong Lin and Liu Chen

January 2001

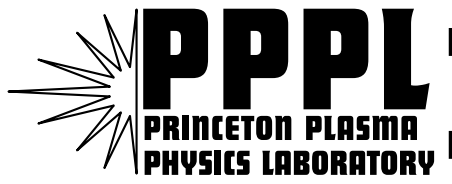

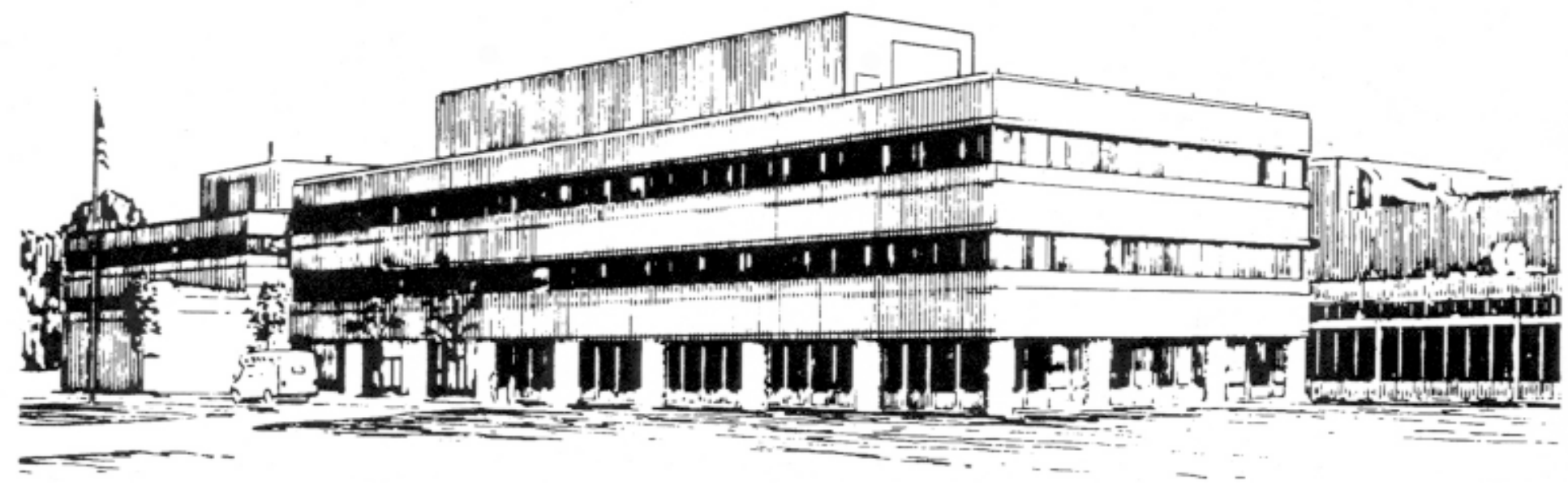

PRINCETON PLASMA PHYSICS LABORATORY PRINCETON UNIVERSITY, PRINCETON, NEW JERSEY 


\section{PPPL Reports Disclaimer}

This report was prepared as an account of work sponsored by an agency of the United States Government. Neither the United States Government nor any agency thereof, nor any of their employees, makes any warranty, express or implied, or assumes any legal liability or responsibility for the accuracy, completeness, or usefulness of any information, apparatus, product, or process disclosed, or represents that its use would not infringe privately owned rights. Reference herein to any specific commercial product, process, or service by trade name, trademark, manufacturer, or otherwise, does not necessarily constitute or imply its endorsement, recommendation, or favoring by the United States Government or any agency thereof. The views and opinions of authors expressed herein do not necessarily state or reflect those of the United States Government or any agency thereof.

\section{Availability}

This report is posted on the U.S. Department of Energy's Princeton Plasma Physics Laboratory Publications and Reports web site in Calendar Year 2001. The home page for PPPL Reports and Publications is: http://www.pppl.gov/pub_report/

DOE and DOE Contractors can obtain copies of this report from:

U.S. Department of Energy

Office of Scientific and Technical Information

DOE Technical Information Services (DTIS)

P.O. Box 62

Oak Ridge, TN 37831

Telephone: (865) 576-8401

Fax: (865) 576-5728

Email: reports@adonis.osti.gov

This report is available to the general public from:

National Technical Information Service

U.S. Department of Commerce

5285 Port Royal Road

Springfield, VA 22161

Telephone: 1-800-553-6847 or

(703) 605-6000

Fax: (703) 321-8547

Internet: http://www.ntis.gov/ordering.htm 


\title{
A Fluid-Kinetic Hybrid Electron Model for Electromagnetic Simulations
}

\author{
Zhihong Lin \\ Princeton Plasma Physics Laboratory, Princeton University, Princeton, NJ 08543 \\ Liu Chen \\ Department of Physics and Astronomy, University of California, Irvine, CA 92697
}

(January 19, 2001)

\begin{abstract}
A fluid-kinetic hybrid electron model for electromagnetic simulations of finite- $\beta$ plasmas is developed based on an expansion of the electron response using the electron-ion mass ratio as a small parameter (Here $\beta$ is the ratio of plasma pressure to magnetic pressure). The model accurately recovers low frequency plasma dielectric responses and faithfully preserves nonlinear kinetic effects (e.g., phase space trapping). Maximum numerical efficiency is achieved by overcoming the electron Courant condition and suppressing high frequency modes. This method is most useful for nonlinear kinetic (particle-in-cell or Vlasov) simulations of electromagnetic microturbulence and Alfvenic instabilities in magnetized plasmas.
\end{abstract}

Magnetic fluctuations in magnetized plasmas have been shown in linear theories to be key ingredients in micro-instabilities [1], drift-Alfven wave instabilities [2], and magnetohydrodynamic instabilities such as toroidal Alfven eigenmodes [3], energetic particle modes [4] as well as the generation of magnetic pulsations in magnetosheres [5]. Nonlinear kinetic study of these electromagnetic fluctuations, meanwhile, is hindered by the difficulty of treating the dynamics of electrons whose characteristic frequency is much faster than that of the low frequency modes of interest. Specifically, the existence of high frequency modes and the electron Courant condition [6] place stringent, unnecessary numerical constraints in nonlinear kinetic simulations. As a result, most turbulence simulations have been focused on the electrostatic limit $[7,8]$. Recently, major efforts have been directed to develop working electron models, e.g., massless electron model (no dissipation) [9] and gyrofluid model (linear closure) [10]. A fully kinetic electron model for gyrokinetic particle simulations has been proposed which extracts out the adiabatic response and solves dynamically only for the nonadiabatic response [11]. Thus, it removes the numerical noise associated with the adiabatic response, which is larger than the nonadiabatic response by the square-root of electron-ion mass ratio. However, the difficulties with high frequency modes and the electron Courant condition remain to be resolved.

In this work, we develop a fluid-kinetic hybrid electron model for nonlinear kinetic simulations of low frequency turbulence in magnetized plasmas. Both the electron response and the perturbed parallel electric field are expanded based on a small electron-ion mass ratio. In the lowest order, the electrons are adiabatic and can be described by fluid equations. Thus, the high frequency modes are removed and no electron Courant condition needs to be observed. In the higher orders, the nonadiabatic response is treated kinetically with all nonlinear kinetic effects preserved. This model combines the good numerical properties of the fluid approach and the accu- rate kinetic effects of the fully kinetic model. It is most useful for nonlinear kinetic (particle-in-cell or Vlasov) simulations of low frequency modes, e.g., shear Alfven waves and ion acoustic waves, in magnetized plasmas.

We consider a shearless slab with uniform magnetic field $\mathbf{B}_{\mathbf{0}}=B_{0} \hat{\mathbf{b}}_{\mathbf{0}}$ and equilibrium uniform Maxwellian ions and electrons,

$$
f_{0}=n_{0}(2 \pi)^{-1 / 2} v_{\alpha}^{-1} e^{-v_{\|}^{2} / 2 v_{\alpha}^{2}}
$$

with $\alpha=i, e$ for ion and electron, respectively, $q_{e}=-e$, $q_{i}=e$, and $v_{\alpha}^{2}=T_{\alpha} / m_{\alpha}$. Assuming the usual gyrokinetic ordering, the gyrokinetic equation [12] for the perturbed distribution function $\delta f_{\alpha}=f_{\alpha}-f_{0}$ is,

$$
\frac{D}{D t} \delta f_{\alpha}=-\frac{D}{D t} f_{0}
$$

where

$$
\frac{D}{D t} \equiv \frac{\partial}{\partial t}+\left(v_{\|} \hat{\mathbf{b}}-\frac{\nabla \phi \times \mathbf{B}}{B^{2}}\right) \cdot \nabla-\frac{q_{\alpha}}{m_{\alpha}} \nabla \psi \cdot \hat{\mathbf{b}} \frac{\partial}{\partial v_{\|}},
$$

$\hat{\mathbf{b}}=\left(\mathbf{B}_{\mathbf{0}}+\delta \mathbf{B}\right) / B$, and $\delta \mathbf{B}=\nabla \times \mathbf{A}_{\|} . \quad k_{\|} \ll k_{\perp}$ is assumed where the wave vector is $\mathbf{k}=k_{\|} \hat{\mathbf{b}}_{\mathbf{0}}+k_{\perp} \hat{\mathbf{k}}_{\perp}$ with $\hat{\mathbf{k}}_{\perp} \cdot \hat{\mathbf{b}}_{\mathbf{0}}=0$. Finite Larmor radius effects in Eq. 1 are omitted for simplicity. The parallel electric field is $E_{\|}=-\nabla \psi$, where

$$
\nabla \psi \cdot \hat{\mathbf{b}}=\nabla \phi \cdot \hat{\mathbf{b}}+\frac{\partial A_{\|}}{\partial t} .
$$

The gyrokinetic Poisson equation in the long wavelength approximation for the electrostatic potential is,

$$
\left(\frac{\rho_{s}}{\lambda_{D}}\right)^{2} \nabla_{\perp}^{2} \phi=-\frac{q_{i} \delta n_{i}+q_{e} \delta n_{e}}{\epsilon_{0}},
$$

where $\lambda_{D}^{2}=\epsilon_{0} T_{e} / n_{0} e^{2}, \rho_{s}=c_{s} / \Omega_{i}, c_{s}=\sqrt{T_{e} / m_{i}}$, $\Omega_{i}=e B_{0} / m_{i}$, and $\delta n_{\alpha}=\int \delta f_{\alpha} d v_{\|}$. The Ampere's law for the vector potential is, 


$$
\nabla_{\perp}^{2} A_{\|}=-n_{0}\left(q_{i} \delta u_{i}+q_{e} \delta u_{e}\right)
$$

with $n_{0} \delta u_{\alpha}=\int v_{\|} \delta f_{\alpha} d v_{\|}$. Equations 1-4 form a complete system governing the electromagnetic fluctuations in our model plasma.

Linearizing Eqs. 1-4 and making the ansatz $e^{i(\mathbf{k} \cdot \mathbf{x}-\omega t)}$, we obtain the linear dispersion relation,

$$
\left(\frac{\omega^{2}}{k_{\|}^{2} v_{A}^{2}}-1\right)\left[1+\zeta_{e} Z\left(\zeta_{e}\right)+\tau+\tau \zeta_{i} Z\left(\zeta_{i}\right)\right]=k_{\perp}^{2} \rho_{s}^{2},
$$

where the $Z$-function is

$$
Z\left(\zeta_{\alpha}\right)=\frac{1}{\sqrt{\pi}} \int \frac{e^{-t^{2}}}{t-\zeta_{\alpha}} d t
$$

with $\tau=T_{e} / T_{i}, \zeta_{\alpha}=\omega / \sqrt{2} k_{\|} v_{\alpha}$ and Alfven speed $v_{A}=B_{0} / \sqrt{\mu_{0} n_{0} m_{i}}$. There are three branches of normal modes in Eq. 5: a kinetic Alfven wave [13] with $\omega=k_{\|} v_{A} \sqrt{1+k_{\perp}^{2} \rho_{s}^{2}}$ for cold ion and adiabatic electron, an ion acoustic wave with $\omega=k_{\|} c_{s}$ for $\tau \gg 1$, and a high frequency mode (gyrokinetic version of the plasma oscillation) [6] with $\omega_{H}=\frac{k_{\|}}{k_{\perp}} \sqrt{\frac{m_{i}}{m_{e}}} \Omega_{i}$ for $\beta_{e} \ll 1$, where the electron beta is

$$
\beta_{e}=\frac{n_{0} T_{e}}{B_{0}^{2} / 2 \mu_{0}} .
$$

The restriction on the time step $\Delta t$ for kinetic simulations of Eqs. 1-4 is imposed by the $\omega_{H}$ mode,

$$
\omega_{H} \Delta t<1,
$$

and the electron Courant condition,

$$
k_{\|} v_{e} \Delta t<1
$$

These time step restrictions are physically unnecessary since the modes of interest (shear Alfven waves and ion acoustic waves) typically have lower frequency $\omega \ll$ $\omega_{H}, k_{\|} v_{e}$. Recognizing that most of electrons behave adiabatically for these low frequency modes, a "splitweight" scheme [11] has been developed for gyrokinetic particle simulations. Instead of solving for the full perturbed distribution function, Eq. 1, this method solves dynamically the kinetic equation governing the nonadiabatic part of the electron response. This is much like the usual analytic formulation [14] and Eqs. 1-4 are solved exactly. Since the perturbed momentum, pressure, and even higher order moments need to be calculated from the electron distribution function which is evolved kinetically, this method has to treat very accurately the dynamics of thermal electrons that contribute predominantly to these moments. Therefore, while it removes the numerical noise associated with the adiabatic response, this scheme still needs to observe [15-17] the time step restrictions of Eqs. 6 and 7, and is subject to the numerical noise of the undamped $\omega_{H}$ mode. Nonetheless, the concept of treating only the nonadiabatic response dynamically does inspire the development of the present hybrid model.

We now formulate a fluid-kinetic hybrid model that can overcome the electron Courant condition and remove the unphysical $\omega_{H}$ mode by solving approximately, rather than exactly, Eqs. 1-4. Noting that the phase velocity of the shear Alfven wave and the ion acoustic wave are typically much smaller than the electron thermal velocity, we can expand the linear solution of Eq. 1 for electron,

$$
\delta f_{e}=\frac{k_{\|} v_{\|}}{k_{\|} v_{\|}-\omega} \frac{e \psi}{T_{e}} f_{0}=\frac{e \psi}{T_{e}} f_{0}\left(1+\frac{\omega}{k_{\|} v_{\|}}+\cdots\right) .
$$

In this expansion, the small parameter $\delta_{m} \ll 1$ is the ratio of the wave phase velocity to the electron thermal velocity, i.e., for the shear Alfven wave,

$$
\frac{\omega}{k_{\|} v_{\|}} \sim \frac{v_{A}}{\sqrt{2} v_{e}}=\left(\frac{m_{e}}{\beta_{e} m_{i}}\right)^{1 / 2} \equiv \delta_{m}
$$

and for the ion acoustic wave, $\delta_{m} \equiv \sqrt{m_{e} / m_{i}}$. $\delta_{m}$ represents the deviation from the adiabatic response. When $\delta_{m}<1$, we can expand Eqs. 1-4 based on $\delta_{m}$ and solve them order by order. Formally, the parallel electric field potential $\psi$ and distribution function $f_{e}$ are expanded,

$$
\begin{gathered}
\psi=\psi^{(0)}+\psi^{(1)}+\cdots, \\
f_{e}=f_{0} e^{\frac{e \psi}{T_{e}}}+\delta g_{e}^{(1)}+\cdots
\end{gathered}
$$

In the lowest order, $\psi=\psi^{(0)}$ and $f_{e}=f_{0} e^{e \psi^{(0)} / T_{e}}$, and all electrons are adiabatic and can be described by a fluid model. In particular, the density is governed by the continuity equation which can be obtained from Eq. 1,

$$
\frac{\partial \delta n_{e}}{\partial t}=-\mathbf{B} \cdot \nabla \frac{n_{0} \delta u_{e}}{B} .
$$

The vector potential evolves according to Eq. 2,

$$
\frac{\partial A_{\|}}{\partial t}=\hat{\mathbf{b}} \cdot \nabla(\psi-\phi) .
$$

These two dynamical equations are closed by field equations, i.e., the Poisson equation,

$$
\left(\frac{\rho_{s}}{\lambda_{D}}\right)^{2} \nabla_{\perp}^{2} \phi=-\frac{q_{i} \delta n_{i}+q_{e} \delta n_{e}}{\epsilon_{0}},
$$

and the Ampere's law,

$$
n_{0} q_{e} \delta u_{e}=-\nabla_{\perp}^{2} A_{\|}-n_{0} q_{i} \delta u_{i} .
$$

The lowest order solution for the parallel electric field is,

$$
e^{\frac{e \psi^{(0)}}{T_{e}}}-1=\frac{\delta n_{e}}{n_{0}} .
$$


Equations 8-12 are a complete set for the fluid electron model in the lowest order in $\delta_{m}$.

In the first order in $\delta_{m}$, we treat the nonadiabatic response using the electron kinetic equation, Eq. 1, with the perturbed field from the lowest order solution,

$$
\frac{D}{D t} \delta g_{e}^{(1)}=f_{0} \frac{e}{T_{e}} e^{\frac{e \psi^{(0)}}{T_{e}}}\left(-\psi_{t}^{(0)}+\frac{\nabla \phi \times \mathbf{B}}{B^{2}} \cdot \nabla \psi\right)
$$

With $\psi_{t}^{(0)}=\partial \psi^{(0)} / \partial t$ which can be evaluated from the lowest order solution of Eqs. 8 and 12,

$$
\frac{e}{T_{e}} \psi_{t}^{(0)}=-\mathbf{B} \cdot \nabla \frac{\delta u_{e}}{B} .
$$

The parallel electric field with the first order correction $\psi=\psi^{(0)}+\psi^{(1)}$ can be obtained from the $f_{e}$ expansion,

$$
e^{\frac{e \psi}{T_{e}}}-1=\frac{\delta n_{e}}{n_{0}}-\frac{\delta n_{e}^{(1)}}{n_{0}}
$$

with $\delta n_{e}^{(1)}=\int \delta g_{e}^{(1)} d v_{\|}$. Equations 13-15 form a complete system for the first order correction of the nonadiabatic response. This procedure can be repeated to achieve accuracy to higher order in $\delta_{m}$.

We now examine the linear dielectric properties of the hybrid electron model with first order accuracy in $\delta_{m}$, Eqs. 8-15. Ion dynamics are treated using the usual gyrokinetic equation, Eq. 1. Linearizing these equations, we obtain the dispersion relation,

$$
\left(\frac{\omega^{2}}{k_{\|}^{2} v_{A}^{2}}-1\right)\left[\frac{1}{1-\zeta_{e} Z\left(\zeta_{e}\right)}+\tau+\tau \zeta_{i} Z\left(\zeta_{i}\right)\right]=k_{\perp}^{2} \rho_{s}^{2} .
$$

As expected, this dispersion relation of the hybrid model is a result of a small parameter expansion of the drift kinetic dispersion relation, Eq. 5,

$$
\frac{1}{1+\zeta_{e} Z\left(\zeta_{e}\right)}=1-\zeta_{e} Z\left(\zeta_{e}\right)+\cdots, \text { for } \zeta_{\mathrm{e}} \sim \delta_{\mathrm{m}} \ll 1 .
$$

Note that the crucial kinetic effect, Landau damping, is retained rigorously in this expansion. This hybrid model can be regarded as a nonlinear closure scheme. Unlike the gyrofluid closure [10] which only treats linear waveparticle interactions, i.e., phase mixing, the hybrid model preserves faithfully nonlinear kinetic effects, e.g., particle trapping by waves. In the limit of small $\delta_{m}$, the hybrid model recovers the exact kinetic results. What is more, it has superior numerical properties as compared to the fully kinetic model. First, there are only two branches of normal modes in Eq. 16: the shear Alfven wave and the ion acoustic wave. The unphysical high frequency $\omega_{H}$ mode is explicitly removed from the hybrid model because of the adiabatic electron response in the lowest order of expansion. Thus, the numerical noise and the time step restriction (Eq. 6) associated with this mode are removed analytically. This results in a lesser required number of particles and a larger time step in the simulations. Secondly, the hybrid model overcomes the electron Courant condition of Eq. 7. In the lowest order with the fluid equations, the time step restriction is the shear Alfven wave or the ion acoustic wave frequency $\omega_{A}$,

$$
\omega_{A} \Delta t<1 \text {. }
$$

In the higher order with kinetic corrections, the time step restriction is the transit time of resonant electrons with a low velocity $v_{\|} \sim v_{A}, c_{s}$. This is identical with the time step of Eq. 17. Therefore, unlike the fully kinetic approach [15-17] where numerical accuracy requires the observation of Courant condition, our hybrid model preserves accurate electron response without the constraint of the Courant condition for both kinetic Alfven wave and ion acoustic wave.

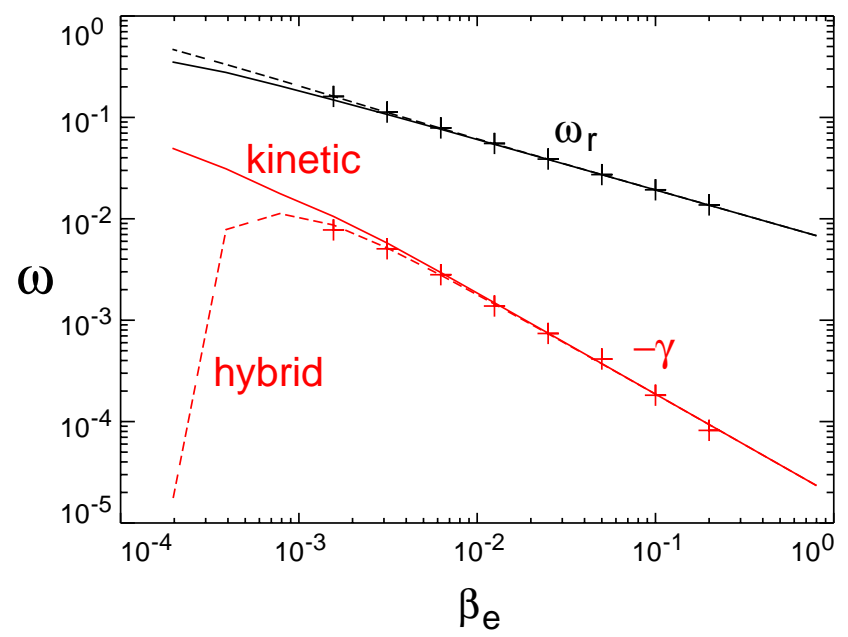

FIG. 1. Kinetic Alfven wave real frequencies $\omega_{r} / \Omega_{i}$ (upper curves) and damping rates $-\gamma / \Omega_{i}$ (lower curves) vs. electron beta. Solid lines are kinetic results, dashed lines are hybrid model results, and "+" points are simulation results.

While the hybrid electron model is valid for any low frequency modes including kinetic Alfven waves and ion acoustic waves, here we study the kinetic shear Alfven wave in a shearless slab to demonstrate the utility of the hybrid model. In these gyrokinetic particle simulations, the electron dynamics is treated using Eqs. 8-15 and the ion contribution is omitted for simplicity (the ion acoustic wave is suppressed). The corresponding analytic dispersion relations of Eqs. 5 and 16 are shown in Fig. 1. The hybrid model results (dashed line) agree with the exact kinetic results (solid lines) for $\beta_{e}>m_{e} / m_{i}$, i.e., $\delta_{m}<1$. The simulation results ("+") also agree very well with the analytic results of the hybrid model. The simulation parameters are $m_{i} / m_{e}=1837, k_{\perp} \rho_{s}=0.4, k_{\|} / k_{\perp}=0.01$, and $0.001<\beta_{e}<0.2$. The number of spatial grids is $N_{g}=64$, the number of electrons is $N_{p}=10000$, and time step is $\omega_{A} \Delta t=0.1$ (which is required for integration of a simple oscillator with frequency $\omega_{A}$ using a second order Runge-Kutta method). For the high- $\beta_{e}$ cases, this 
time step violates the electron Courant condition of Eq. 7 and the grid size is also larger than the collisionless electron skin depth, yet accurate results are obtained. Thus the issue of resolving the skin depth in a fully kinetic model $[16,17]$ is removed from our hybrid model.

The nonlinear simulation result for $\beta_{e}=0.0125$ is shown in Fig. 2. The amplitude of the magnetic perturbation is oscillatory (solid line) due to the trapping of resonant electrons. The bounce frequency $\omega_{b}$ in Fig. 2 is close to the theoretical estimate of $\omega_{b}=k_{\|} v_{e} \sqrt{e \psi / T_{e}}$ (in the simulation $\left.e \psi / T_{e}=0.0025\right)$. At the minimum of the field perturbation, the electron distribution function also exhibits population inversion around the resonant point. In an otherwise identical linear simulation (dashed line) the magnetic perturbation decays exponentially. The observation of particle trapping in the nonlinear simulation shows that the hybrid model faithfully preserves this important nonlinear kinetic effect.

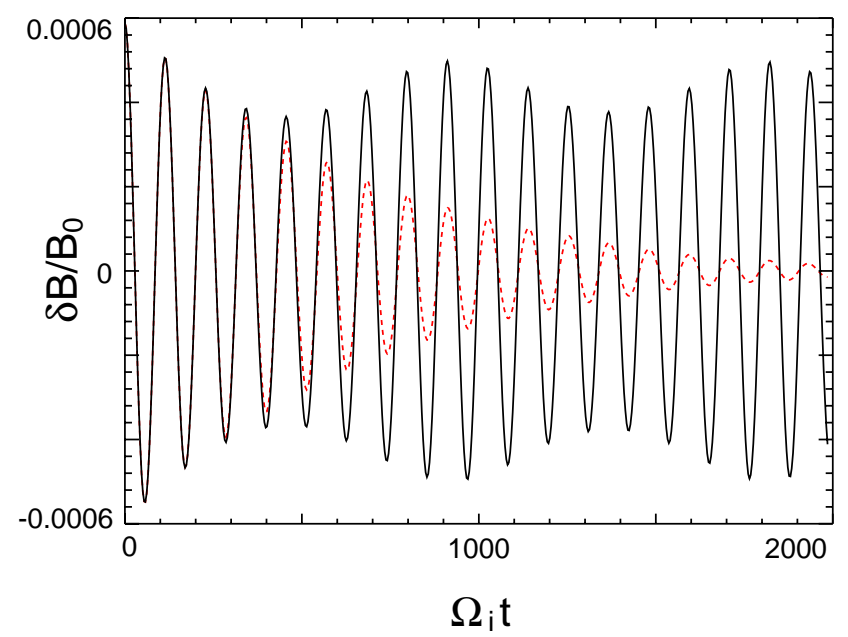

FIG. 2. Nonlinear simulation of kinetic Alfven wave exhibits oscillation in amplitude of perturbed magnetic field (solid line), while linear simulation shows exponential decay (dashed line).

It is clear from Fig. 1 that the electromagnetic version of the hybrid electron model is not valid for $\beta_{e} \leq m_{e} / m_{i}$. In this regime, the shear Alfven wave has a phase velocity faster than the electron thermal velocity. Since simulations need to resolve the fast time scale of this inertial Alfven wave anyway, electrons do not place any additional numerical constrain. Therefore, a conventional fully kinetic treatment is warrant. In such low beta plasmas, we can also extend the hybrid model to the electrostatic limit which is of more practical interest. Here, the magnetic perturbation is negligible in most cases because the fluctuating current is very small and electrostatic fluctuations dominate the dynamics. Thus for $\beta_{e} \leq m_{e} / m_{i}$, simulations can be reduced to an electrostatic one (effectively $\beta_{e} \sim 0$ ). Here the smallness parameter is the ratio of sound speed to electron thermal speed, $\delta_{m} \equiv \sqrt{m_{e} / m_{i}}$. In the lowest order in $\delta_{m}$, electrons are adiabatic and we only need Eq. 10. The next order nonadiabatic correction is governed by Eq. 13. As expected, the linear dispersion relation of this electrostatic system has only one normal mode: the ion acoustic wave. The unphysical $\omega_{H}$ mode, which has a thermal fluctuation level much higher than that of the ion acoustic wave [6], is again explicitly removed. Furthermore, the electron Courant condition can be violated. These are the advantages of the hybrid model in the electrostatic limit as compared to the electrostatic split-weight scheme [11] which is fully kinetic.

This Work is supported by U.S. DOE contract number DE-AC02-76CHO3073 (PPPL) and grant number DEFG03-94ER54271 (UC Irvine), NSF Grant ATM-9971529 (UC Irvine), and by the DOE Plasma Sciences Advanced Computing Initiative. Z. Lin acknowledges helpful discussions with W. M. Tang and W. W. Lee.

[1] W. M. Tang, Nuclear Fusion 18, 1089 (1978).

[2] F. Zonca, L. Chen, J. Q. Dong, and R. A. Santoro, Phys. Plasmas 1, 1517 (1999).

[3] C. Z. Cheng, L. Chen, and M. S. Chance, Ann. Phys. 161, 21 (1985).

[4] L. Chen, Phys. Plasmas 1, 1519 (1994).

[5] L. Chen and A. Hasegawa, J. Geophy. Res. 96, 1503 (1991).

[6] W. W. Lee, J. Comput. Phys. 72, 243 (1987).

[7] Z. Lin, T. S. Hahm, W. W. Lee, W. M. Tang, and R. B. White, Science 281, 1835 (1998).

[8] A. M. Dimits, G. Bateman, M. A. Beer et al., Phys. Plasmas 7, 969 (2000).

[9] G. Zhao and L. Chen, Bull. Am. Phys. Soc. 44, 302 (1999).

[10] P. Snyder, Ph.D Thesis, Princeton University, 1999.

[11] I. Manuilskiy and W. W. Lee, Phys. Plasmas 7, 1381 (2000).

[12] T. S. Hahm, W. W. Lee, and A. Brizard, Phys. Fluids 31, 1940 (1988).

[13] A. Hasegawa and L. Chen, Phys. Fluids 19, 1924 (1976).

[14] E. A. Frieman and L. Chen, Phys. Fluids 25, 502 (1982).

[15] W. W. Lee, J. L. Lewandowski, Z. Lin, and T. S. Hahm, Bull. Am. Phys. Soc. 45, 193 (2000).

[16] Y. Chen and S. Parker, Bull. Am. Phys. Soc. 45, 159 (2000).

[17] B. I. Cohen, A. M. Dimits, and W. M. Nevins, Bull. Am. Phys. Soc. 45, 193 (2000). 


\section{External Distribution}

Plasma Research Laboratory, Australian National University, Australia

Professor I.R. J ones, Flinders University, Australia

Professor J oão Canalle, Instituto de Fisica DEQ/IF - UERJ , Brazil

Mr. Gerson O. Ludwig, Instituto Nacional de Pesquisas, Brazil

Dr. P.H. Sakanaka, Instituto Fisica, Brazil

The Librarian, Culham Laboratory, England

Library, R61, Rutherford Appleton Laboratory, England

Mrs. S.A. Hutchinson, JET Library, England

Professor M.N. Bussac, Ecole Polytechnique, France

Librarian, Max-Planck-Institut für Plasmaphysik, Germany

J olan Moldvai, Reports Library, MTA KFKI-ATKI, Hungary

Dr. P. Kaw, Institute for Plasma Research, India

Ms. P.J . Pathak, Librarian, Insitute for Plasma Research, India

Ms. Clelia De Palo, Associazione EURATOM-ENEA, I taly

Dr. G. Grosso, Instituto di Fisica del Plasma, Italy

Librarian, Naka Fusion Research Establishment, J AERI, J apan

Library, Plasma Physics Laboratory, Kyoto University, J apan

Research Information Center, National Institute for Fusion Science, J apan

Dr. O. Mitarai, Kyushu Tokai University, J apan

Library, Academia Sinica, Institute of Plasma Physics, People's Republic of China

Shih-Tung Tsai, Institute of Physics, Chinese Academy of Sciences, People's Republic of China

Dr. S. Mirnov, Triniti, Troitsk, Russian Federation, Russia

Dr. V.S. Strelkov, Kurchatov Institute, Russian Federation, Russia

Professor Peter Lukac, Katedra Fyziky Plazmy MFF UK, Mlynska dolina F-2, Komenskeho Univerzita, SK-842 15 Bratislava, Slovakia

Dr. G.S. Lee, Korea Basic Science Institute, South Korea

Mr. Dennis Bruggink, Fusion Library, University of Wisconsin, USA

Institute for Plasma Research, University of Maryland, USA

Librarian, Fusion Energy Division, Oak Ridge National Laboratory, USA

Librarian, Institute of Fusion Studies, University of Texas, USA

Librarian, Magnetic Fusion Program, Lawrence Livermore National Laboratory, USA

Library, General Atomics, USA

Plasma Physics Group, Fusion Energy Research Program, University of California at San Diego, USA

Plasma Physics Library, Columbia University, USA

Alkesh Punjabi, Center for Fusion Research and Training, Hampton University, USA

Dr. W.M. Stacey, Fusion Research Center, Georgia Institute of Technology, USA

Dr. J ohn Willis, U.S. Department of Energy, Office of Fusion Energy Sciences, USA

Mr. Paul H. Wright, Indianapolis, Indiana, USA 
The Princeton Plasma Physics Laboratory is operated by Princeton University under contract with the U.S. Department of Energy.

\author{
Information Services \\ Princeton Plasma Physics Laboratory \\ P.O. Box 451 \\ Princeton, NJ 08543
}

Phone: 609-243-2750

Fax: 609-243-2751

e-mail: pppl_info@pppl.gov

Internet Address: http://www.pppl.gov 